\title{
16 channel receiver with 20 GHz Ge Photodiodes
}

\author{
J.M. Fedeli ${ }^{\text {a }}$ L.Virot ${ }^{\mathrm{a}, \mathrm{b}, \mathrm{d}}$, J.M. Hartmann ${ }^{\mathrm{a}}$, P.Grosse ${ }^{\mathrm{a}}$, W.Bogaerts ${ }^{\mathrm{c}}$, L. Vivien ${ }^{\mathrm{b}}$ \\ ${ }^{a}$ CEA, LETI, Minatec Grenoble, France \\ ${ }^{b}$ IEF, CNRS, Université Paris-Sud XI, Orsay, France \\ ${ }^{c}$ Photonic Research Group,- Ghent University, Ghent, Belgium \\ ${ }^{d}$ ST Microelectronics, Crolles, France
}

\begin{abstract}
A $200 \mathrm{GHz} 16$ channel receiver with polarization management was obtained with a 2D grating coupler, 2xAWGs and $16 \mathrm{Ge}$ photodiodes. PDL was below $1 \mathrm{~dB}, \mathrm{BW}$ above $20 \mathrm{GHz}$, receiver sensitivity in the order of $0.08 \mathrm{~A} / \mathrm{W}$.
\end{abstract}

\section{DEMONSTRATOR FOR HELIOS PROJECT}

Submicron silicon photonics have generated an increasing interest in recent years, mainly for optical telecommunications or for optical interconnects in microelectronic circuits. The rationale of silicon photonics is the reduction of the cost and energy of communications systems through the integration of photonic components and an electronic integrated circuit (IC) on a common chip (telecommunications applications), or the enhancement of IC performances with the introduction of optics inside a high performance chip (core to core communications), or low cost sensors. By co-integrating optics and electronics on the same chip, high- functionality, high-performance and highly integrated devices can be fabricated with a well-mastered microelectronics fabrication process. The FP7 HELIOS project aims to combine a photonic layer with a CMOS circuit by using microelectronics fabrication processes. A first goal was to develop high performance generic building blocks for a broad range of applications: WDM sources by III-V/Si heterogeneous integration [1], fast modulators [2,3] and detectors [4], passive circuits and packaging. With these building blocks, different demonstrators are assembled. One of those is a $16 \times 10 \mathrm{~Gb} / \mathrm{s}$ transceiver@1550nm made of a transmitter with hybrid lasers and silicon modulators, which will be reported elsewhere and a receiver, which is the subject of the present communication.

This receiver is sketched in Figure 1: A 2D surface grating couples the light coming from a single mode fiber SMF fiber into the circuit and separates the two polarizations while transforming the TM polarization into TE. Identical $200 \mathrm{GHz} 16$ channel AWGs receive the two input signals and demultiplexe the guided TE modes. The two 16 output waveguides are then connected to 16 Ge photodiodes.

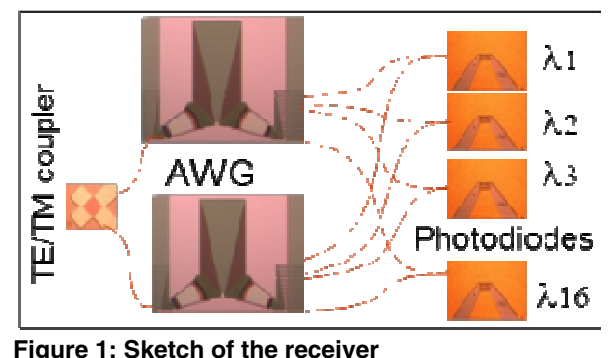

\section{PASSIVES AND GE PHOTODETECTORS}

We have developed a self self-aligned process for the fabrication of the waveguides using two photolithography steps with a $193 \mathrm{~nm}$ stepper and two Si dry etching steps for the fabrication of gratings and waveguides on optical SOI substrates from SOITEC (220nm Si on top of $2 \mu \mathrm{m}$ Buried OXide). The process starts with the deposition of a 100nm High Temperature Oxide (HTO) layer (that will serve as a hardmask) on top of the silicon layer. The gratings and the waveguide arms are first patterned, followed by Reactive Ion Etching of silica with C4F8, defining the hardmask. The quality of the edges is given by the resist roughness and by the hard mask etching process. The silicon is then partially etched $(65 \mathrm{~nm})$ with $\mathrm{HBr}$ gazes. The resulting thickness is controlled by spectroscopic ellipsometry in order to precisely define the grating teeth depth. In the second lithography step, gratings are protected by the resist and the remaining hardmask is used for the self-aligned etching of the waveguides. Then a full silicon etch down to the box completes the waveguide fabrication. A $10 \mathrm{~nm}$ thin thermal oxide (consuming $\sim 5 \mathrm{~nm}$ silicon) was then employed to smooth the waveguide sidewalls. We then defined cavities for the selective epitaxial growth of Germanium. This is achieved by deposition of a silica layer which is etched at the end of waveguides. In order to achieve direct coupling, the silicon floor of the cavities is etched down to 50nm on top of the BOX. Germanium was then selectivity grown in the cavities and Chemical Mechanical Polishing used to adjust the thickness. A thick layer of silica was then deposited and windows opened in it down to the Ge layer underneath. In a self-aligned process, the 
doped regions ( $\mathrm{N}$ and $\mathrm{P}$ ) of the lateral PIN Ge photodetector were then defined by ion implantation of Phosphorus and Boron in the defined openings. The separation of the openings thus defines the width of the Ge intrinsic region. A $1 \mu \mathrm{m}$ thick $\mathrm{SiO} 2$ layer was then deposited and planarized before etching $400 \mathrm{~nm}$ diameter holes in it. These holes were filled with Ti/W and planarized in order to get $\mathrm{W}$ vias. A Ti/TiN/AlCu metal stack was deposited on this flat surface. DUV 248nm lithography together with and Cl2 etching was used to fabricate the metallic pads. (Figure 3).

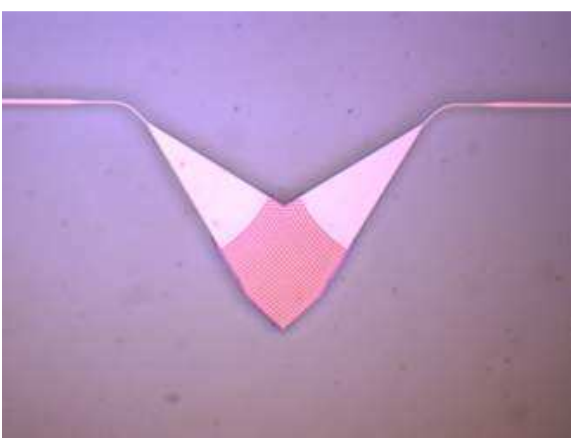

Figure 2: 2D grating coupler

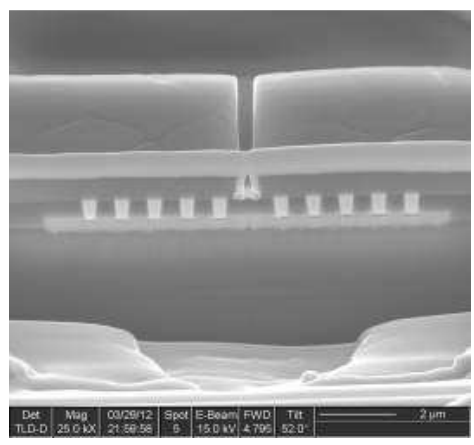

Figure 3: Cross-sectional image of a Ge photodiode with $\mathrm{W}$ plugs and $\mathrm{Al}$ pads (a localized W layer was deposited during the FIB operation on top of the metal pads)

The tests were performed on a $200 \mathrm{~mm}$ wafer prober. Using basic spirals, the propagation losses were statistically measured on the wafers. For $480 \mathrm{~nm}$ x $220 \mathrm{~nm}$ cladded waveguides, the losses were found at $2.3 \mathrm{~dB} / \mathrm{cm}$. The $2 \mathrm{D}$ grating coupler was adapted from the Gent University design [5] to the self-aligned technology (Figure 2). Using 2x 2D couplers mounted face to face, the Polarization Dependant Loss (PDL), the spectral response could be measured. The optimal efficiency for the $2 \mathrm{D}$ grating coupler was experimentally found to be $15 \%$ ( 8dB coupling losses) at $1550 \mathrm{~nm}$ with a $3 \mathrm{~dB}$ bandwidth of $55 \mathrm{~nm}$. The minimum PDL was measured at $\sim 1 \mathrm{~dB}$ at $\sim 1550 \mathrm{~nm}$. The 16 channels AWG with $200 \mathrm{GHz}$ separation is also adapted from a design from Gent University [5] with the technology and with the introduction of some absorbing sections. To reduce the crosstalk, an enlarged strip waveguide is used for the array part. So the crosstalk levels were measured at $-15 \mathrm{~dB}$, and the minimum center-channel insertion losses around $2.8 \mathrm{~dB}$.

The Ge photodiode is a butt coupled PIN lateral type of $10 \mu \mathrm{m}$ length. The photodiode sensitivity $\sim 0.8 \mathrm{~A} / \mathrm{W}$. Capacitance is in the $10 \mathrm{fF}$ range and dark current of the order of $20 \mathrm{nA}(-0.5 \mathrm{~V})$ as seen on figure 4 . The typical photodiode was connected to a 2D grating coupler and the polarization of the incoming was randomly changed. Upon the separation between the doped region, the bandwith can be adjusted up to $90 \mathrm{GHz}$ [4]. For this $10 \mathrm{~Gb} / \mathrm{s} \mathrm{demonstrator}$, a separation of $1 \mu \mathrm{m}$ was selected and the measured bandwidth is shown upon the reverse voltage on Figure 5 . With an applied voltage of $-1 \mathrm{~V}$, the bandwidth of all dies is above $15 \mathrm{GHz}$ while the mean value is around $20 \mathrm{GHz}$ which is comfortable for $10 \mathrm{~GB} / \mathrm{s}$ operation and should be also enough for $25 \mathrm{Gbit} / \mathrm{s}$ operation in new receivers.

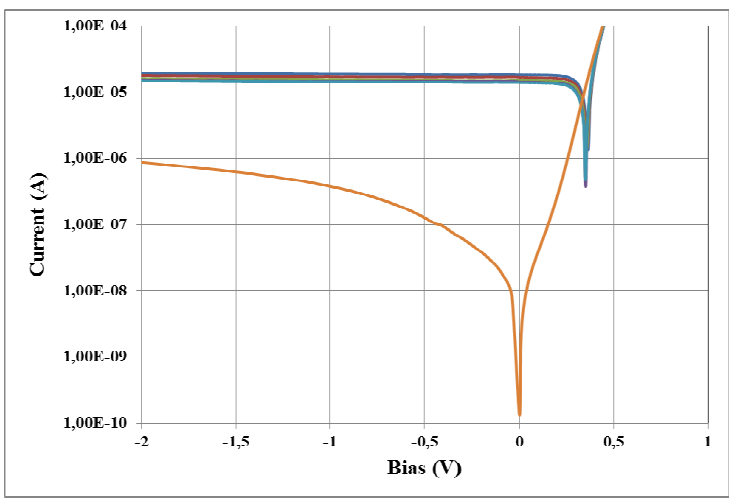

Figure 4: Idark and Photocurrent for different polarizations of the Ge photodiodes

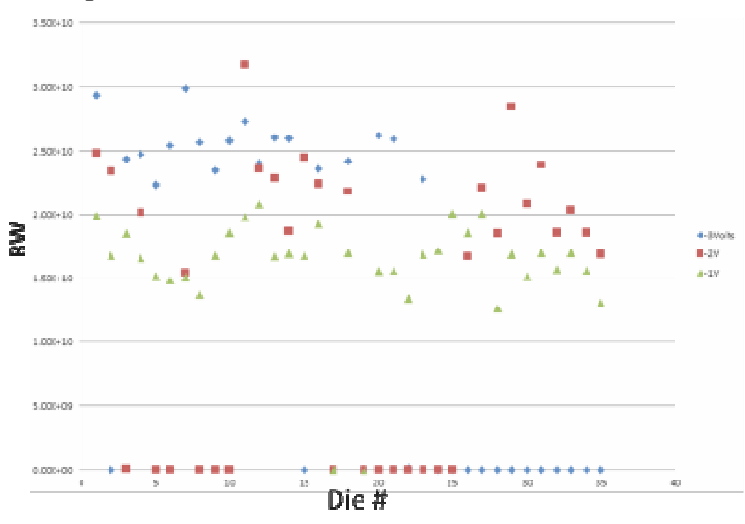

Figure 5: Bandwidth of the Ge photodiodes

\section{RECEIVER}

For demonstration of a 16 channel receiver, a circuit shown on Figure 6 was assembled with the different building blocks. At the left part, a set of input 2D gratings couplers was connected to the inputs of the two identical AWG. Only the centered input grating was coupled to a SMF. Sixteen germanium photodetectors (length $10 \mu \mathrm{m}$ and width $1 \mu \mathrm{m}$ ) 
were disposed in an array with Ground Signal Ground electrodes. Each is connected on one side to one output of the upper AWG and on the other side to the bottom AWG by butt coupling.

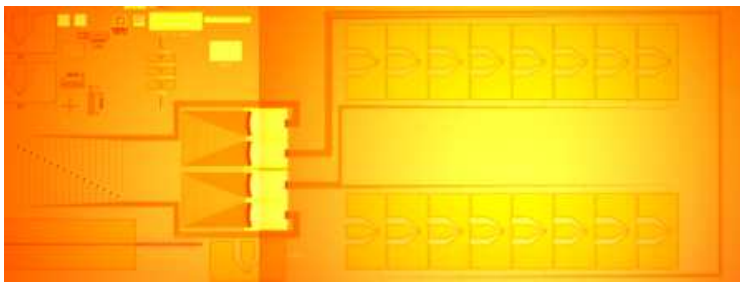

Figure 6: Top-view Optical Microscopy of the 16 channel receiver with 2D couplers (left) and Ge PD (right)

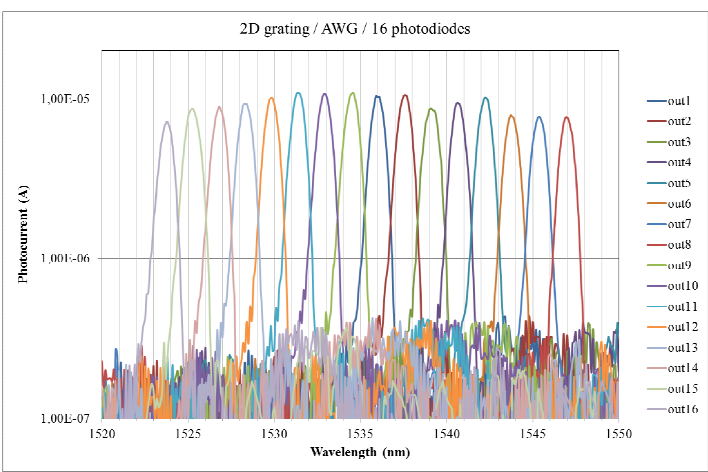

Figure 7: Photodiodes measurement after AWG

The spectral characteristic of the receiver is shown in figure 7. With the losses and sensitivity of the basic blocks, the overall receiver sensitivity is in the order of $0.08 \mathrm{~A} / \mathrm{W}$ with a channel separation of $1.6 \mathrm{~nm}$, corresponding to 200GHz. The 2D grating coupler is responsible for the highest loss of sensitivity and should be optimized. Other solutions such as inverse taper or $1 \mathrm{D}$ grating coupler with separation of TE and TM coupled to a polarization rotator could be more efficient.

\section{CONCLUSION}

This receiver demonstrates the capability of silicon photonics for producing high speed WDM receivers. The Ge photodiodes can reach bandwidths exceeding the capability of Trans Impedance Amplifier. Improvement in the sensitivity requires some optimization of the optical coupling.

\section{AKNOWLEDGMENT}

The research leading to these results has received funding from the European Community's Seventh Framework Programme (FP7/2007-2013) under grant agreement $n^{\circ} 224312$ HELIOS.

\section{REFERENCES}

[1] M. Lamponi, S. Keyvaninia, C. Jany, F. Poingt, F. Lelarge, G. de Valicourt, G. Roelkens, D. Van Thourhout, S. Messaoudene, J.-M. Fedeli, G.H. Duan, "Low-threshold heterogeneously integrated InP/SOI laser with a double adiabatic taper coupler", IEEE Photonics Technology Letters, Volume: 24, Page(s): 76 - 78, 2012.

[2] D. J. Thomson, F. Y. Gardes, Y. Hu, G. Mashanovich, M. Fournier, P. Grosse, J-M. Fedeli, and G. T. Reed, "High contrast 40Gbit/s optical modulation in silicon," Opt. Express 19, 11507-11516 (2011)

[3] M.Ziebell \&al, "40 Gbit/s low-loss silicon optical modulator based on a pipin diode", to appear in Optics Express in 2012

[4] L.Vivien \& al, "Zero-bias 40Gbit/s germanium waveguide photodetector on silicon," Opt. Express 20, 1096-1101 (2012)

[5] W. Bogaerts, D. Taillaert, P. Dumon, D. Van Thourhout, R. Baets, and E. Pluk, "A polarization-diversity wavelength duplexer circuit in silicon-on-insulator photonic wires.,” Optics express, vol. 15, no. 4, pp. 1567-78, Feb. 2007. 